\title{
Blue rubber bleb nevus syndrome associated with tuberous sclerosis complex and CNS involvement
}

\author{
Hajar Alreef, MBBS, Mashael AlKhateeb, MD.
}

\begin{abstract}

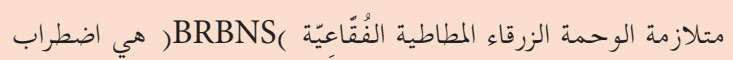

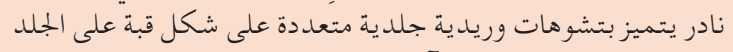

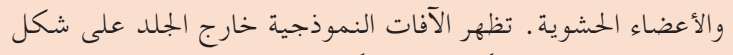

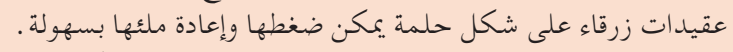

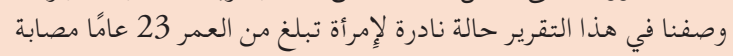
BRBNS.

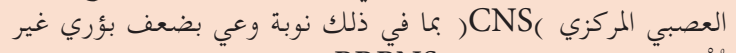

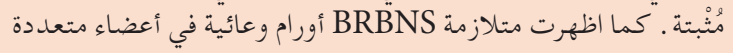

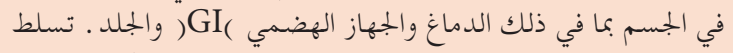

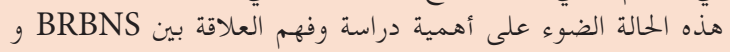
TSC
\end{abstract}

Blue rubber bleb nevus syndrome (BRBNS) is a rare disorder that is characterized by multiple domeshaped cutaneous venous malformations on the skin and visceral organs. Typical extra-cutaneous lesions have the appearance of blueish nipple-shaped nodules that can easily compress and refill. We described a rare case of a 23-year-old female with BRBNS and tuberous sclerosis complex (TSC) that presented with central nervous system (CNS) involvement including unprovoked focal impaired awareness seizure. Her BRBNS presents with hemangiomas involving multiple organs in the body including the brain, gastrointestinal (GI) system, and skin. This case highlights the importance of studying and understanding the association between BRBNS and TSC as it may lead to improved understanding

Neurosciences 2021; Vol. 26 (2): 207-211 doi: 10.17712/nsj.2021.2.20200111

From the Department of Neuroscience, King Faisal Specialist Hospital and Research Centre, Riyadh, Saudi Arabia

Received 3rd August 2020. Accepted 3rd October 2020

Address correspondence and reprint request to: Dr. Hajar Alreefi, Alfaisal University, College of Medicine, Riyadh, Saudi Arabia. E-mail: halreefi@alfaisal.edu

ORCID ID: https://orcid.org/0000-0002-3268-043X
First described in 1860, blue rubber bleb nevus syndrome (BRBNS) is a disorder characterized by multiple dome-shaped cutaneous venous malformations on the skin and visceral organs. ${ }^{1}$ The BRBNS is an extremely rare disorder with only approximately 200 cases being reported since the term was coined. ${ }^{1}$ Typical extra-cutaneous lesions have the appearance of blueish nipple-shaped nodules that can easily compress and refill. ${ }^{2}$ These lesions frequently affect the gastrointestinal (GI) system and cause hemorrhage, iron-deficiency anemia, intussusception, and volvulus. ${ }^{3}$ In addition to GI involvement, these lesions have been shown to affect the central nervous system and may cause fatal hemorrhage. ${ }^{2}$ Herein, we report a case of BRBNS in a patient with TSC and CNS involvement that resulted in focal impaired awareness seizures.

Case Report. Patient information and clinical findings. A 23-year-old female presented to the clinic with a history of focal impaired awareness seizure. She is a known case of tuberous sclerosis (confirmed by genetic testing and evident on MRI brain) and BRBNS, which the patient was diagnosed with early in childhood. Her BRBNS presents with hemangiomas involving multiple organs in the body including the brain, GI system, and skin.

Diagnostic assessment and therapeutic intervention. Earlier in her diagnosis, she was managed with various modalities, such as chemotherapy and interventional radiology, which failed to control her disease and specifically her active GI bleeding. To treat her subsequent iron-deficiency anemia, she was transfused on a weekly basis. Owing to her progressive uncontrolled disease, she traveled to seek further treatment at medical centers in the United States, where her disease was

Disclosure. The authors declare no conflicting interests, support or funding from any drug company. 
controlled by surgical resection and interventional sclerotherapy. The patient returned to Saudi Arabia and was later commenced on sirolimus, which she showed some response to. In 2016, the patient started developing multiple new lesions on her neck and trunk. Later, she started presenting complaints that are consistent with focal aware seizures. These attacks are characterized by loss of memory over a couple of minutes that is slowly regained in the proceeding 30 min. The patient reports feeling fear that lasts seconds to minutes preceding the seizure. It has been reported that her first attack happened when she was 2 years old. It occurred daily at any time during the day or night. After 9 years of age, it became less frequent and occurred once per week in the form of staring and behavioral arrest with mouth automatism lasting less than 10 seconds. At that time, she had been started initially on ethosuximide and valproic acid. After her 20th birthday, the semiology changed to focal impaired awareness seizure presenting in the form of palpitations and fear followed by behavioral arrest lasting less than 30 seconds. Carbamazepine was effective at that time in controlling her seizures as a mono-therapy. Alas, at the age of 23 years, the seizure recurred. At that time, the immunosuppressant for her dermatological case, sirolimus, was commenced and had interacted with the carbamazepine she was on. Therefore, levetiracetam (LEV) was added and was later reported to have aggravated her anxiety symptoms. Our patient was then shifted to lamotrigine (LTG) due to the psychiatric side effects she developed from LEV including anxiety and depression, which has successfully kept her seizure free during her 6-months follow up.

Follow-Up and outcomes. Upon examining the patient, she had small, dark blue, round or ellipsoid, soft compressible nevi (20-mm diameter) on her face, abdomen, and back. Her physical exam and vital signs were otherwise within normal limits. Upon further investigation, a 30-min EEG study performed was found to be normal with rhythmic mid-temporal theta bursts of drowsiness (RMTD), a benign variant also known as the psychomotor variant. Video-EEG or continuous EEG to ensure the absence of seizures is yet to be performed. Multisequential multiplanar nonenhanced and enhanced MRIs of the head and neck were performed showing extensive scalp, neck, chest wall as well as para-spinal soft tissue mass lesions involving also deep regions like the deep neck and chest, posterior deep cervical, oral, around the thyroid and para-tracheal regions. Additionally, the radiological findings showed a similar soft tissue lesion in the right oropharynx causing narrowing. The overall appearance was described as suggestive of extensive multiple vascular malformations in keeping with the known diagnosis of Blue Rubber Bleb Nevus Syndrome. Other MRI findings have shown to be consistent with Tuberous Sclerosis of multiple bilateral cortical/subcortical tubers involving bilateral frontal, parietal as well as left temporal lobes with ill-defined gray-white matter junction and cortical thickening.

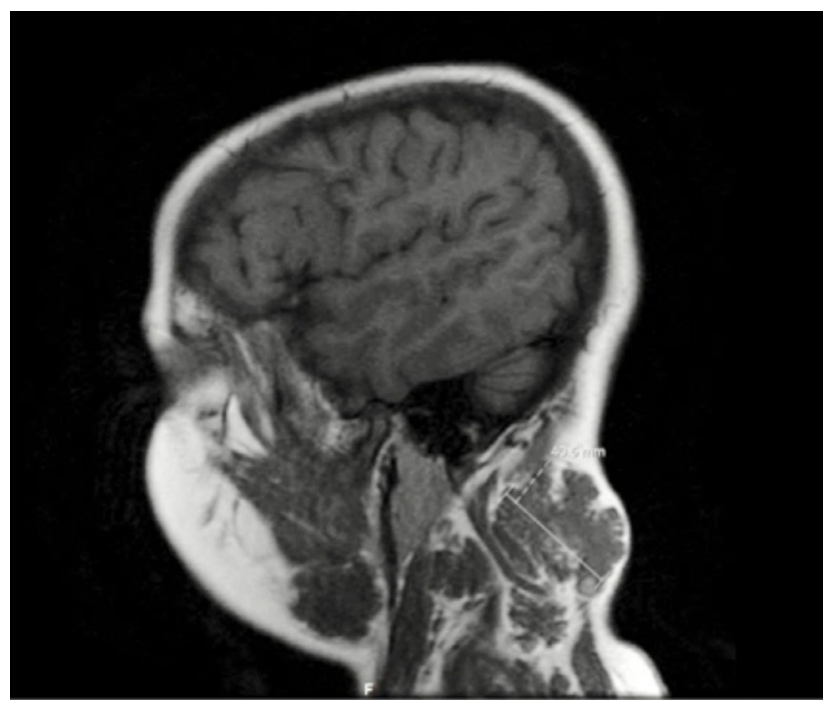

Figure 1 - Magnetic resonance imaging (MRI) T1 of head and neck sagittal section showing extensive vascular hemangioma of the posterior neck.

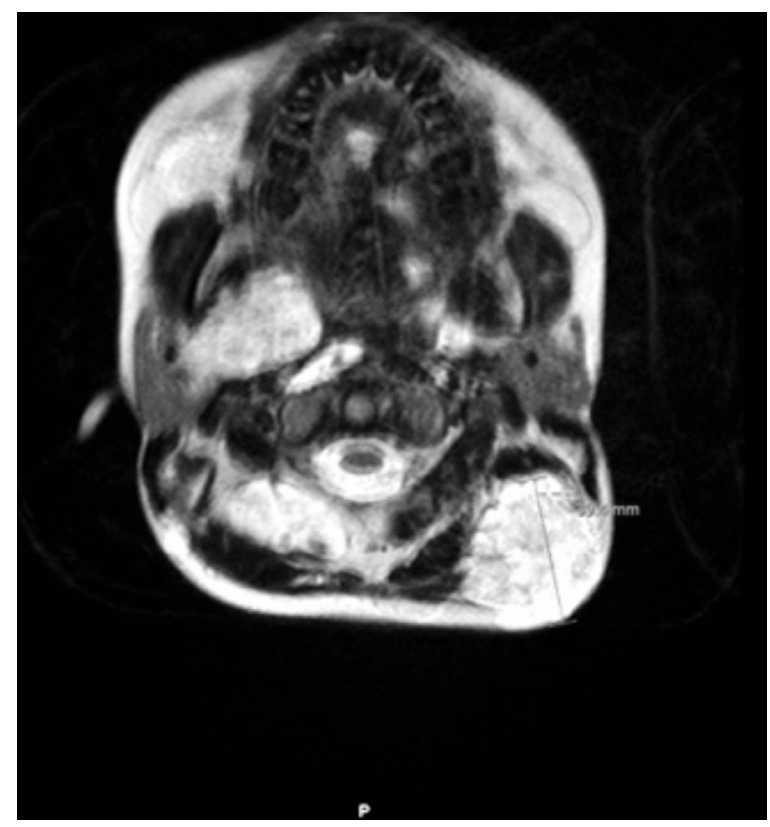

Figure 2 - Magnetic resonance imaging (MRI) T1 of head and neck transverse section showing extensive para-spinal soft tissue mass lesions. 


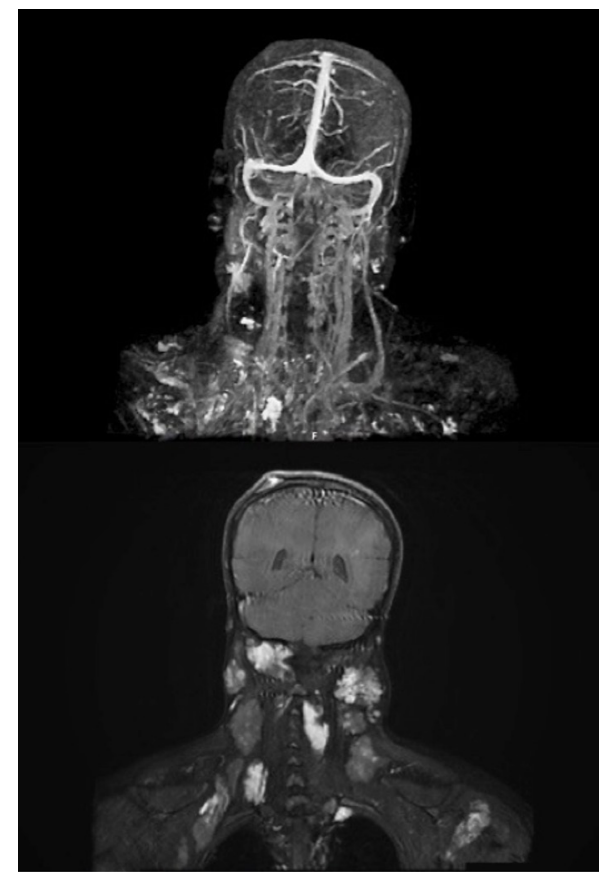

Figure 3 - Post-contrast coronal MR of the head and neck shows extensive scalp, neck, upper chest and posterior para-spinal lobulated T2 hyper-intense soft tissue mass lesions suggestive of soft tissue venous malformation.

Discussion. The BRBNS with CNS involvement is rare and has been seldom reported. Some of the diagnoses in the reported cases were not clear. To date, 6 cases have been described including the case that has been thoroughly documented by Waybright et al. ${ }^{2}$ In 1978, Waybright et al. have described a BRBNS patient who presented with focal seizures and lateralized neurologic signs. The possible neurologic outcomes of BRBNS can present in multiple ways depending on the size and location of the cerebral angiomas. ${ }^{5}$ Owing to the heterogeneity of CNS manifestations and the risk of developing angioma thrombosis, it is typically challenging to evaluate the prognosis of the disease. Thus, it is essential for patients to undergo strict monitoring and thorough neurologic evaluation. ${ }^{5}$ However, to date, there have been no documented cases of BRBNS associated with TSC and subsequent CNS involvement. The patient in this study had multiple cutaneous venous malformations, bilateral cortical/ subcortical tubers, slight prominence of the ventricular system without evidence of acute hydrocephalus, and multiple hemangiomas in the brain, scalp, oropharynx, and chest. The diagnosis was confirmed on the basis of typical skin lesions and other clinical findings. For GI lesions, angiography, endoscopy, and MRI are favorable

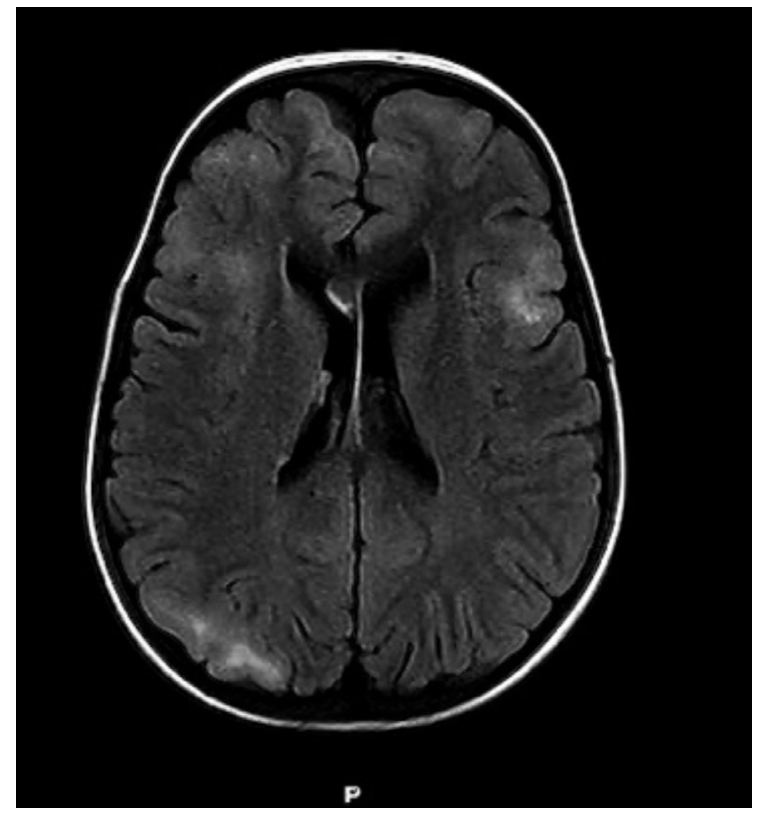

Figure 4 - Axial FLAIR of the brain demonstrates multiple bilateral cortical/subcortical tubers involving bilateral frontal parietal as well as left temporal lobes with ill-defined gray-white matter junction and cortical thickening.

for diagnosis. In cases of CNS involvement, further imaging studies (e.g., CT and MRI) have been described as useful noninvasive tools. In addition, MRI has been described as a useful tool for screening asymptomatic patients. ${ }^{2}$ Typically, the treatment is symptom- and patient-based. This includes modalities to manage the bleeding and transfusion therapy. A combined lasersteroid therapy for GI hemangiomas has been reported by some investigators. The BRBNS cases are mostly sporadic in nature; familial cases have been recently associated with somatic mutations in TEK, the gene encoding TIE2, the endothelial cell tyrosine kinase receptor for the angiopoietins. These cases appear to be inherited in an autosomal dominant pattern. The TIE/angiopoietin family are closely implicated in and responsible for processes driving angiogenesis, be it in the physiological or in the pathological setting. The TIE2 activation integrates several intracellular signaling pathways, including the phosphati-dylinositol-3-kinase (PI3K)/AKT pathway promoting endothelial cell survival. PI3K in turn stimulates downstream regulation of mTOR. ${ }^{3}$ Similarly, TSC1/2 complex, the tumor suppressor genes responsible for Tuberous Sclerosis Complex, stimulates upstream activation signals from growth factor receptors via the pathway of (PI3K)/ AKT. The mechanisms by which the TSC $1 / 2$ complex is regulated and in turn controls mTOR activity are 


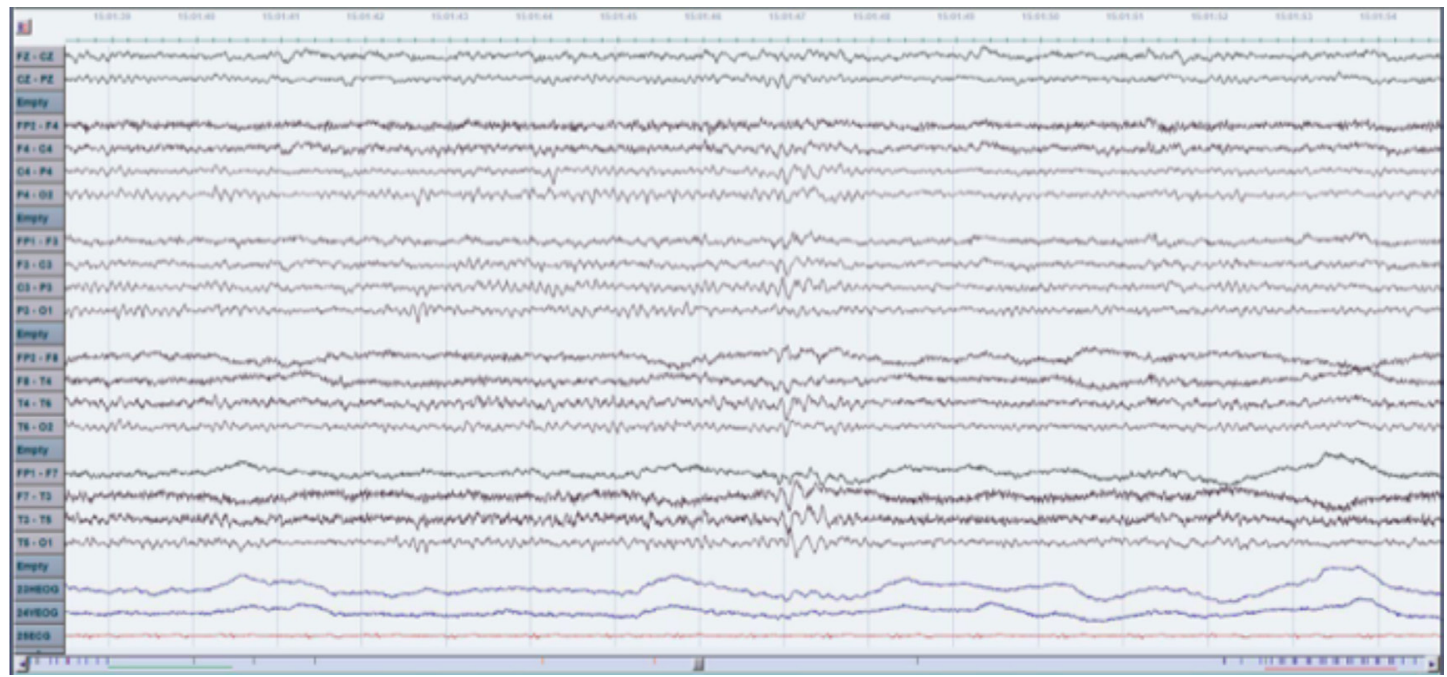

Figure 5 - 30-Minute EEG results showing rhythmic midtemporal theta bursts of drowsiness.

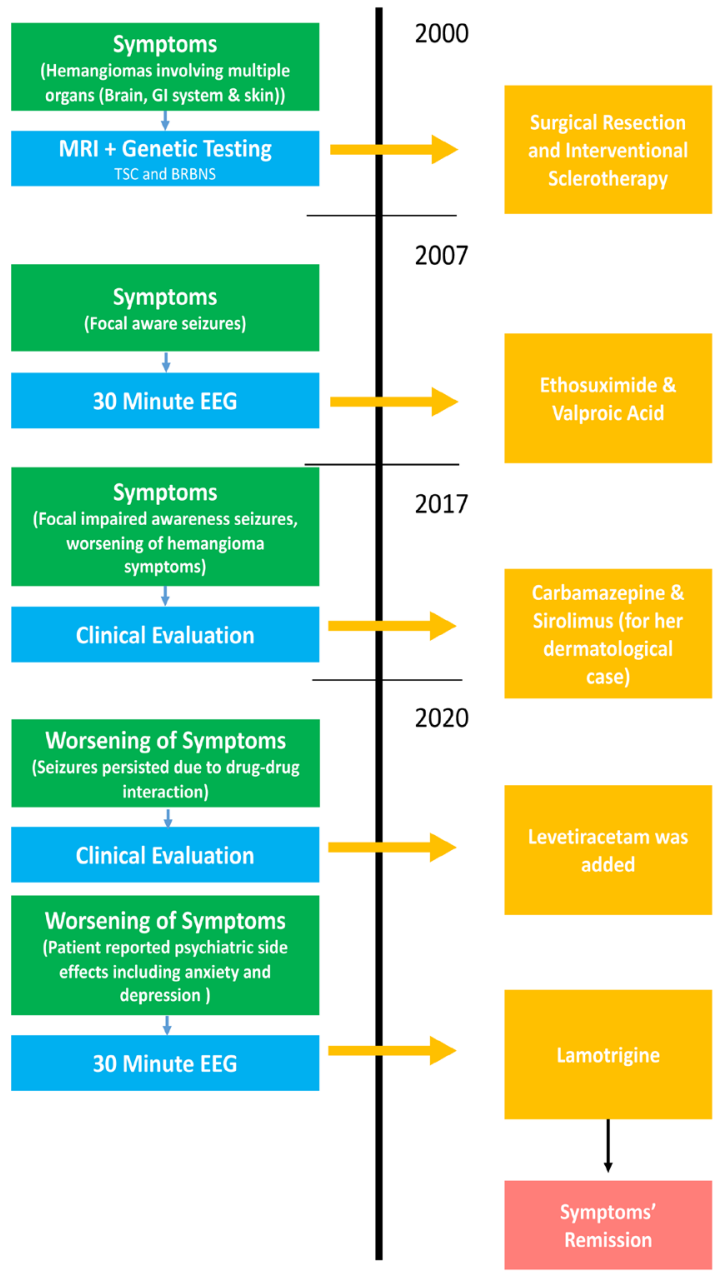

Figure 6 - Case report timeline. merely beginning to be unraveled. ${ }^{4}$ Tuberous sclerosis complex is an autosomal genetic disorder characterized by the development of several benign tumors in the body. Vascular malformations or abnormalities in the setting of TSC are well-described in scientific literature. Angiomyolipomas are most commonly described, followed by arterial malformations, which are considerably less common. According to previous publications, most patients with TSC develop epilepsy, and most develop multiple seizure types. It has been reported that the most frequent seizure types in TSC are focal seizures and focal spasms. ${ }^{7}$ In the current case, our patient first presented with seizures at the age of 2 years. Her most recent seizures presented as focal impaired awareness seizures described as loss of memory over a couple of minutes that is preceded by feeling of fear lasting between seconds to minutes. Typically, seizure onset occurs in the first year of life, yet adults remain at risk. Because TSC-associated epilepsy usually presents early in life, seizure semiology often reflects the locations of cortical tubers and can potentially affect functional maturation. ${ }^{3}$ Focal seizures or infantile spasms frequently present in TSC patients with early onset epilepsy. ${ }^{7}$ In regards to the fact that drug-resistant epilepsy in TSC is common, many patients achieve full seizure control despite the presence of lesions in the brain. ${ }^{6}$ Studies conducted show that focal rhythmic slow wave activity, predominant on the temporal region without diffusion, was the typical reported pattern in TSC patients presenting with focal epilepsy. ${ }^{7}$ In the contrary, our patient presented with a normal 30-minute EEG study displaying rhythmic mid- 
temporal theta bursts of drowsiness (RMTD). RMTDs are generally considered benign variants with uncertain significance; however, concerns have been raised for their ictal potential. ${ }^{8}$ They were later confirmed to be the least epileptogenic of temporal discharges. ${ }^{8}$ However, generalized spike and wave discharges may be completely absent during wakefulness in some patients, making routine EEG studies yield a low sensitivity for identifying interictal epileptiform discharges (IEDs). It is safe to say that long-term video-EEG monitoring is the gold standard to confirm the diagnosis, classification and localization of epileptic seizures. ${ }^{9}$ A study by X. Liu et $\mathrm{a}^{10}$ concluded that the first-hour sleep EEG is able to reliably predict the occurrence of IEDs during long-term video-EEG recording and can be utilized as a time-efficient method to identify patients with IEDs, mitigating the burden in the adult epilepsy monitoring unit. Guidelines suggest that antiepileptic drugs (AEDs) are indicated as the first treatment option and shall follow those of other epilepsies. ${ }^{4}$ The presence of BRBNS in the setting of TSC has never been documented before. Moreover, it is unclear whether the patient's epileptiform activity is a result of her BRBNS manifesting with cerebral hemangiomas or TSC cortical tubers, yet it has been evident that compliance with the selected mono-therapeutic antiepileptic medication has been beneficial in seizure control. Nevertheless, our study is limited by a number of factors including its retrospective and single case nature, the atypical presentation of TSC and the absence of a long-term video-EEG recording. Additional genetic mutations or complex pathways may be involved and require further characterization. Despite the limitations, the present case highlights the importance of studying the associations between BRBNS and TSC as it may lead to improved understanding of combined pathways and the further development of preferable management plans for patients.

In conclusion, our patient diagnosed with BRBNS and TSC in her childhood demonstrated a rare presentation of focal impaired awareness seizure. Her BRBNS presents with hemangiomas involving multiple organs in the body including the brain, GI system and skin. We report this case to illustrate the importance of studying and understanding the association between these two diseases as it may lead to improved understanding of combined pathways.
Patient's perspective. The patient reports that she feels relieved to have her seizures in control. Moreover, she is glad that the previous psychiatric side effects including anxiety and depression have remitted after the drug switch to lamotrigine. She reports noticing major improvement in her quality of daily life.

Acknowledgment. We would like to sincerely thank Dr. Khalid Omar Alahmadi, for his review of the radiology images. We would also like to thank Falcon Scientific Editing [http://www. https://falconediting.com/] for editing and reviewing this manuscript for English language.

\section{References}

1. Nahm WK, Moise S, Eichenfield LF, Paller AS, Nathanson L, Malicki DM, Friedlander SF. Venous malformations in blue rubber bleb nevus syndrome: variable onset of presentation. $J$ Am Acad Dermatol 2004; 50: S101-S106.

2. Kim SJ. Blue rubber bleb nevus syndrome with central nervous system involvement. Pediatr Neurol 2000; 22: 410-412.

3. Wong XL, Phan K, Rodriguez Bandera AI, Sebaratnam DF. Sirolimus in blue rubber bleb naevus syndrome: A systematic review. J Paediatr Child Health 2019; 55: 152-155. doi: $10.1111 /$ jpc. 14345 .

4. Chu-Shore CJ, Major P, Camposano S, Muzykewicz D, Thiele EA. The natural history of epilepsy in tuberous sclerosis complex. Epilepsia 2010; 51: 1236-1241.

5. Eirís-Puñal J, Picón-Cotos M, Viso-Lorenzo A, Castro-Gago M. Epileptic disorder as the first neurologic manifestation of blue rubber bleb nevus syndrome. J Child Neurol 2002; 17 : 219-222.

6. Song J, Swallow E, Said Q, Peeples M, Meiselbach M, Signorovitch J, et al. Epilepsy treatment patterns among patients with tuberous sclerosis complex. J Neurol Sci 2018; 391: 104-108.

7. Savini MN, Mingarelli A, Vignoli A, Briola FL, Chiesa V, Peron A, et al. Ictal signs in tuberous sclerosis complex: Clinical and video-EEG features in a large series of recorded seizures. Epilepsy Behav 2018; 85: 14-20.

8. Kang JY, Krauss GL. Normal Variants Are Commonly Overread as Interictal Epileptiform Abnormalities. J Clin Neurophysiol 2019; 36: 257-263.

9. Shih JJ, Fountain NB, Herman ST, Bagic A, Lado F, Arnold $S$, et al. Indications and methodology for videoelectroencephalographic studies in the epilepsy monitoring unit. Epilepsia 2018; 59: 27-36.

10. Liu X, Issa NP, Rose S, Wu S, Sun T, Towle LV, et al. The first-hour-of-the-day sleep EEG reliably identifies interictal epileptiform discharges during long-term video-EEG monitoring. Seizure 2018; 63: 48-51. 